myself, in a very extensive experience of this operation, has ever seen it before. Otitis in its various forms is not uncommon, especially in those children who have had previous ear trouble (in this case no history of any ear trouble could be obtained). It is, I think, generally due to sepsis spreading up the Eustachian tubes from the raw surface left round the orifices by the scraping. I am sure I have seen this complication less frequently since I have adopted the plan of scraping only strictly in the middle line and leaving the few scraps around the orifices of the tubes alone. Another important factor in the avoidance of subsequent ear trouble is the after-treatment, $a$ week in bed in a warm room being the most essential point. Secondly, it brings home closely to one the fact that this operation is not always the trifle it is sometimes thought to be. Many complications may follow it-e.g., ear trouble, suppuration in the lymph glands of the neck, hæmorrhage which may even be fatal, and so on. I think we should never tell the parents that the operation is a very minor one.

I am largely indebted to my colleagues, Mr. G. Morgan and Mr. H. H. Taylor, for advice and assistance in connexion with this case.

Brighton.

\section{ORAL SEPSIS IN ITS CONNEXION WITH THROAT DISEASE. ${ }^{1}$}

BY GEORGE IRVINE STEWART, M.A., B.Sc., M.B., C.M. ABERD., F.R.C.S. ENG.,

COUNTY SCHOOL MEDICAL OFFICER, EAST SUFFOLK; LATE CHIEF ASSISTANT, THROAT DEPARTMENT, ST. THOMAS'S HOSPITAL.

\section{The Signs of Oral Sepsis.}

THERE are many more than are to be found in text-books. All of them can be categorised under the signs of inflammation of mucous membranes-redness, swelling, heat, pain, increased secretion, and loss of function. A peritonsillar abscess is the best example of an intense oral sepsis.

Redness. -This varies in intensity and can be general or local ; locally it is most evident opposite a focus such as a septic tooth. In slight cases it is evident as a red line parallel to, but separated by one-sixteenth of an inch from, the border of the gums. As long as this red line is present there is oral sepsis. It is most persistent on the gums bordering the lower molars, and particularly the lower incisors. A characteristic reddening from oral sepsis is to be seen on the soft palate, as a red band half an inch broad commencing on the anterior pillar and arching round towards the centre of the soft palate, but just stopping short of the uvula. The remainder of the soft palate may be quite pale and the red band shows up very distinctly.

Swelling. - This is evident in most cases. It involves all the mucous membranes-of the gums; of the cheeks, which have the teeth plotted out in the odematous swelling; of the tongue, which is similarly marked by the teeth; and of the lips. The swelling of the lips is most marked in infants, leading to a great protrusion and eversion of the lower lip.

Heat and pain are not extreme, but in some cases the breath appears hotter than normal, and discomfort causes the speech to be slovenly.

Increased secretion is evidenced in slight cases by the breaking of bubbles between the back part of the tongue and the soft palate when the mouth is opened. In the worst cases-e.g., in peritonsillar abscess-the dribbling is excessive. Dribbling is also well seen in the "teething" of infants, which is neither more nor less than a fairly intense stomatitis, and is most evident in bottle-fed babies in whom decomposition of milk is more likely to occur.

Loss of funotion.-Of all the signs of oral sepsis this is the most marked and has been the least recognised in its significance and its detrimental effects. It is characterised by diminution or loss of function of all the muscles of the oropharynx-viz., those of the pharynx, palate, tongue, cheeks, and lips. The buccal, oral, and lingual muscles are used for forwarding the food particles to the gullet, for keeping the mouth clear of food, for cleansing the mouth and teeth, and with the pharynx for articulation of speech. The teeth owe a great part of their cleanliness to proper tonicity of those muscles, and the moment their function is diminished by oral sepsis there is an increase of deposited muco-pus and an increase of oral sepsis; that is to say, a true vicious circle is started. The teeth that receive the least cleaning naturally can be readily identified-viz., the lower incisors and the lower molars, precisely the ones that show the red line, the sign of oral sepsis, longest.

The speech in oral sepsis, on account of the lack of tone in the muscles and from the discomfort caused by the swelling of the mucous membranes, becomes slovenly, partially guttural in character, the back part of the tongue being used in preference. It is so characteristic that perhaps the term "circumvallate" speech might be coined for this, as it is in the region of the circumvallate papillæ that the speech is articulated. An extreme case of this sort in a child where the speech was almost unintelligible was cured by proper use of the toothbrush (and the exercises in articulation by the teacher which before had been tried in vain).

The result of this loss of function of the muscles, to which I should wish to call most particular attention, is the dropping of the lower jaw and of the lower lip, which becomes thereby everted. The well-known open-mouth vacant expression usually attributed to adenoids and other nasal obstruction is really a characteristic of oral sepsis, which itself may be set up by nasal obstruction. The socalled "adenoid facies" is really that of oral sepsis, however induced. It is well seen in "teething " infants.

\section{The Effect of Oral Sepsis on the Throat Generally and Particularly on the Tonsils.}

My attention was first drawn to this by the need of finding other causes for laryngitis than "hysterical aphonia" and "nasal laryngitis." In throat hospitals the practice prevailed of at once depressing the tongue with the spatula, thereby concealing the teeth from view, without previously examining the state of the mouth. The practice of first drawing the cheeks aside to examine the state of the teeth is getting more and more universal. The following cases helped to emphasise the importance of oral sepsis in throat work.

CAsE 1. - A male, aged 40 years, diagnosed by a wellknown laryngologist as having tuberculous laryngitis. The cords were reddened and there was much muco-pus on the slightly swollen arytenoids. Quiescent tuberculosis was present at the right apex. He had a complete upper tooth plate lying on a row of septic stumps, each surrounded by florid granulations. Removal of the stumps on my advice cured the laryngitis in a month.

CAsE 2.-A male, aged 19 years. His voice began to break four years ago. For the past two years he had been under treatment at a throat hospital for "squeaky" voice. The cords were reddened; approximation was incomplete. Removal of one stump and careful brushing of the teeth produced in six weeks such an alteration in the voice that his family and himself were continuously laughing about it. He had now a clear bass voice.

OASE 3. - The patient, a male medical practitioner, used to suffer from four or five severe colds every winter. He got severe septic stomatitis caused by sucking a post-mortem wound of the finger. Great attention has since been paid to cleansing the teeth. He now has one "cold" in two years on the average.

Seven elementary school teachers frequently off duty for loss of voice, quinsies, and sore-throat; since having the teeth attended to they have rarely any trouble.

Peritonsillar abscess.-Of 49 cases of this condition 47 had an extremely septic lower molar on the same side. One case (bilateral) had septic molars on both sides. The pus from those abscesses has the same characteristic odour as that from a dental antrum. The oral sepsis in those cases is extreme and is usually regarded as secondary ; it is probably primary.

Unilateral enlargement of the tonsil. - of 231 cases (in children of school age) of unilateral enlargement of the tonsil 135 had dental caries, mostly of lower molars, and oral sepsis on the side of the enlarged tonsil ; 67 had dental caries and oral sepsis on both sides; 16 had no oral sepsis at all ; and 15 had dental caries and oral sepis on the opposite side. I have never seen a case of unilateral inflammatory enlargement of the tonsil in an infant, in whom, of course, the oral sepsis is always bilateral. I believe that the organisms round septic teeth travel directly backwards along the sulci on either side of the lower jaw, and, meeting with 
the tonsil, settle there and cause its inflammatory enlargement. This would also explain the peculiar position of the red band of oral sepsis on the soft palate mentioned above.

\section{Physiological Function of the Ionsil and other Adenoid} Tissue in the Pharynx.

In children the lymphadenoid tissue is more extensive than in the adult and is spread out on the wall of the pharynx, forming a complete ring continuous with the adenoid tissue in the naso-pharynx. The lower limit of adenoid tissue is very irregularly defined and may in cases reach the glottis. As age increases this adenoid tissue disappears and remains chiefly in the tonsil and the so-called lateral bands of the pharynx. It is possible that this disappearance is the result of the frequent attacks of catarrhal conditions in children, such as measles, scarlet fever, sore-throats and common colds, \&c. Each attack would cause a degeneration of certain portions of adenoid tissue which would not be replaced. I would look upon the adenoid tissue in the pharynx as a protective barrier in which the attacking micro-organisms are dealt with actively, in which leucocytosis is most active and where immunisation of the body can be developed. Every attack of the invading micro. organisms would cause an inflammatory enlargement of the adenoid tissue, and in the fight certain portions of the adenoid tissue would be killed, so that the power of the adenoid tissue to resist invasion would be diminished by each attack. In addition, however, the numerous crypts existing in adenoid tissue act as good covering for the attacking organisms where they mass together for a further onslaught, which is rendered the more easy on account of the lessened resistance already produced, and once the adenoid tissue is severely degenerated it is not only useless as a protecting barrier but provides a point of vantage for further invasion.

The tonsil itself I would look upon as the special adenoid (lymphatic) gland of the mouth, lying openly in it, specialised it is true, but just in the same way that a Peyer's patch in the intestine is specialised. A tonsillitis would thus be an adenitis, and a peritonsillar abscess a periadenitis. As a rule the tonsil and other adenoid tissue in the pharynx act as sufficient scavengers for the organisms in the mouth, and at the most there is simply inflammatory enlargement caused. But occasionally the tonsil and adenoid tissue are overwhelmed in the attack and the next link in the lymphatic chain becomes involved-viz., the glands at the angle of the jaw - and these take up the fight, undergoing enlargement in the process. If the attack is still more severe then the organisms no longer confine themselves to the mouth, but travel either upwards to the naso-pharynx or downwards to the stomach and lungs. Naturally when the tonsils and other adenoid tissue are already badly degenerated and act as harbourages for micro-organisms, the barrier power is extremely diminished and attacks of nasopharyngitis and bronchitis become more and more frequent. I believe that the spread of inflammatory conditions from the oro-pharynx to the naso-pharynx by continuity is more frequent than is supposed. It must be remembered that Greville MacDonald long ago pronounced the nasal cavity to be normally free from organisms, while the mouth is normally crowded with them.

4. Ireatment and Indioations for Such Based upon the Above.

When inflammatory enlargement of the tonsils is recognised the treatment is that of enlarged glands in any part of the body: 1. Remove the cause. 2. If that is not sufficient, then the enlarged degenerated tonsil should be removed by surgical interference. Removal of the cause is the prevention or cure of oral sepsis, the extraction of septic molars, and careful mechanical cleansing of the teeth by brushing. This must precede surgical removal of the tonsil. In fact, the reckless way in which we all have removed tonsils with. out attending to the causal oral sepsis cannot be too strongly condemned. It is to my mind the explanation of why so many cases show unpleasant complications and bad results. Removal of the tonsils would be less indicated by their actual increase in size than by the evidence of their inefficiency, as shown by swelling of the glands at the angle of the jaw and by frequent attacks of naso-pharyngitis and of bronchitis. The use of the tooth-brush is essential before, during, and after tonsillotomy, and as a means of preventing attacks upon, and preserving the integrity of, the adenoid protecting ring cannot be too strongly adrocated.

Ipswich.

\section{A CASE OF PRIMARY SARCOMA OF THE DUODENUM.}

BY H. M. MACKENZIE, M.B. EDIN., D.P.H. CANTAB., CAPTAIN, I.M.S. ; PROFESSOR OF PATHOLOGY, MEDICAL COLLEGE, LAHORE, INDIA.

THE comparatively rare occurrence of primary sarcomatous growths originating in the small intestine, and more especially in the duodenum, lends some degree of interest to the present case.

The patient, a Hindu labourer, aged 18 years, was admitted to the Mayo Hospital, Lahore, on Dec. 21st, 1908, under the care of Major H. G. Melville, I.M.S., complaining of pain in the abdomen and back and swelling of the feet. His family and personal history were good and up to the date of the present illness his health had always been fair. The present illness began some 12 days before admission, when he first felt pain in the abdomen and back, and some days later he noticed swelling of his feet. No treatment was adopted, but as the symptoms became more severe he came to hospital. On admission the patient, an anæmic, poorly developed youth, was suffering from pain in the abdomen, especially marked in the right hypochondriac and right lumbar regions and also from pain in the back in the middle line, the pain in both places being increased on pressure. He was markedly jaundiced and passed clay-coloured stools, but there was no diarrhœa or melæna. The urine was normal. The appetite was diminished and thirst was increased and the temperature was subnormal. On examination of the abdomen a large, moderately firm mass, moving with respiration and mobile on palpation, was found to occupy the right hypochondriac, right lumbar, and part of the epigastric and umbilical regions, as shown in the illustration, the inner margin being

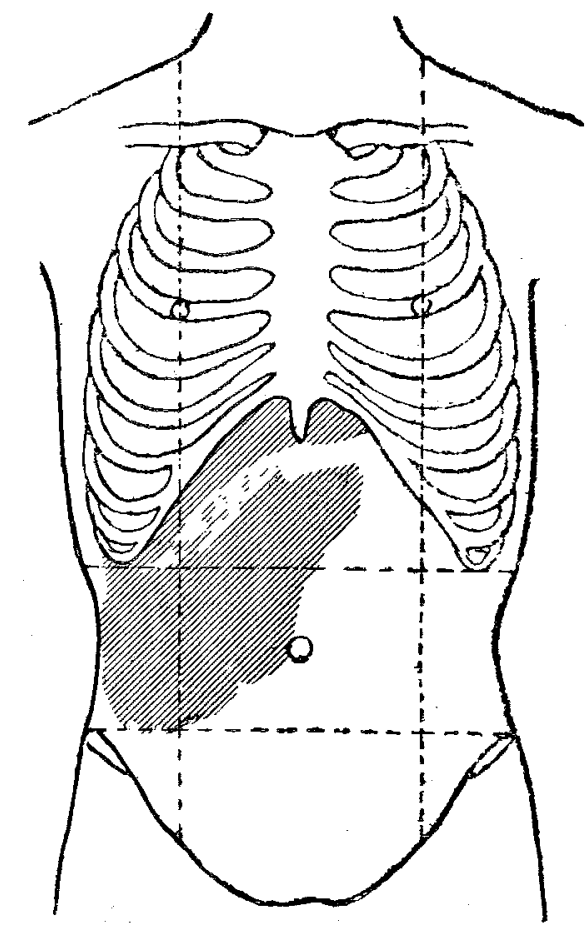

distinctly nodular. The lower edge of the liver was palpable in parts, other parts being somewhat obscured, and light percussion gave a tympanitic note between the upper margin of the swelling and the edge of the liver, except on the right side where the dulness was continuous. There was some fluctuation of the swelling towards the upper and inner margin. The mass was diagnosed as a malignant growth of the right kidney, probably sarcomatous, with dilatation of the gall. bladder from obstruction of the common bile duct. General symptomatic treatment was adopted, but the condition of the patient steadily grew worse and he died on Jan. 12th, 1909.

The post-mortem examination was conducted 24 hours after death. The body was that of an emaciated, poorly developed youth and showed very marked jaundice. Postmortem lividity was slight and rigor mortis was present in the lower limbs only. The abdomen, which was retracted, showed slight fulness on the right side but no other abnormality externally. On opening the 